\title{
Transcriptome sequencing of a chimaera reveals coordinated expression of anthocyanin biosynthetic genes mediating yellow formation in herbaceous peony (Paeonia lactiflora Pall.)
}

Daqiu Zhao, Yao Jiang, Chuanlong Ning, Jiasong Meng, Shasha Lin, Wen Ding and Jun Tao*

\begin{abstract}
Background: Herbaceous peony (Paeonia lactiflora Pall.) is a traditional flower in China and a wedding attractive flower in worldwide. In its flower colour, yellow is the rarest which is ten times the price of the other colours. However, the breeding of new yellow P. lactiflora varieties using genetic engineering is severely limited due to the little-known biochemical and molecular mechanisms underlying its characteristic formation.

Results: In this study, two cDNA libraries generated from P. lactiflora chimaera with red outer-petal and yellow inner-petal were sequenced using an Illumina HiSeq 2000 platform. 66,179,398 and 65,481,444 total raw reads from red outer-petal and yellow inner-petal cDNA libraries were generated, which were assembled into 61,431 and 70,359 Unigenes with an average length of 628 and 617 nt, respectively. Moreover, 61,408 non-redundant All-unigenes were obtained, with 37,511 All-unigenes (61.08\%) annotated in public databases. In addition, 6,345 All-unigenes were differentially expressed between the red outer-petal and yellow inner-petal, with 3,899 up-regulated and 2,446 down-regulated All-unigenes, and the flavonoid metabolic pathway related to colour development was identified using the Kyoto encyclopedia of genes and genomes database (KEGG). Subsequently, the expression patterns of 10 candidate differentially expressed genes (DEGs) involved in the flavonoid metabolic pathway were examined, and flavonoids were qualitatively and quantitatively analysed. Numerous anthoxanthins (flavone and flavonol) and a few anthocyanins were detected in the yellow inner-petal, which were all lower than those in the red outer-petal due to the low expression levels of the phenylalanine ammonialyase gene (PIPAL), flavonol synthase gene (PIFLS), dihydroflavonol 4-reductase gene (PIDFR), anthocyanidin synthase gene (PIANS), anthocyanidin 3-O-glucosyltransferase gene (PI3GT) and anthocyanidin 5-O-glucosyltransferase gene (PI5GT).

Conclusion: Transcriptome sequencing (RNA-Seq) analysis based on the high throughput sequencing technology was an efficient approach to identify critical genes in P. lactiflora and other non-model plants. The flavonoid metabolic pathway and glucide metabolic pathway were identified as relatived yellow formation in P. lactiflora, PIPAL, PIFLS, PIDFR, PIANS, PI3GT and PI5GT were selected as potential candidates involved in flavonoid metabolic pathway, which inducing inhibition of anthocyanin biosynthesis mediated yellow formation in P. lactiflora. This study could lay a theoretical foundation for breeding new yellow P. lactiflora varieties.
\end{abstract}

Keywords: Anthocyanins, Flower colour, Flavonoids, Herbaceous peony, Transcriptome, Yellow

\footnotetext{
*Correspondence: taojun@yzu.edu.cn

Key Laboratory of Crop Genetics and Physiology of Jiangsu Province, College of Horticulture and Plant Protection, Yangzhou University, Yangzhou 225009, Jiangsu, P.R. China
} 


\section{Background}

With the rapid development of the national economy and increasing integration in the international community, the demand for flowers is changing from high yield to high quality in recent years. Among the many qualities that influence flowers, flower colour is one of the most important indicators that determines its ornamental quality. Ornamental plants with novel flower colours can effectively stimulate the senses and psychology of consumers, as well as have a broader market appeal [1], such as the blue rose (Rosa rugosa Thunb.) and yellow violet (Matthiola incana $\mathrm{R}$. Br.) [2]. Thus, breeding new flower cultivars with rare colours has been an important target for flower breeders.

Herbaceous peony (Paeonia lactiflora Pall.) is a traditional flower in China that belongs to the Paeoniaceae family and has been a well-known symbol of prosperity. In China, it enjoys a high status as a traditional flower and has shared the name with "The king and minister of flowers" with the tree peony (Paeonia suffruticosa Andr.) [3]. Until recently, there has been a large germplasm resource of $P$. lactiflora with more than 600 cultivars worldwide, and the flower colour characteristics vary widely among different cultivars, which can be divided into nine flower colour categories, i.e., red, pink, white, blue, purple, green, yellow, black and double colour [4]. Among these flower colours, yellow, which represents gold, suggests riches and honour, as well as reveals the king demeanour in flowers. In addition, the commercial price of yellow P. lactiflora cultivars is usually approximately 10 times more than purple or red cultivars. Whereas, yellow cultivars of P. lactiflora are very rare, where currently, there is only one pure yellow cultivar, namely 'Huangjinlun' [5]. This limitation can not highly meet the needs of consumers. Thus, breeding new $P$. lactiflora cultivars with yellow flowers has been an important aim, although it has been challenging using selective breeding and hybridisation breeding. Therefore, understanding of the biochemical and molecular mechanisms of yellow characteristic formation in P. lactiflora, identifying its key genes, and the ability to modify flower colour using modern genetic engineering are important to breed new $P$. lactiflora varieties in the future. However, very limited genomic resources are available for $P$. lactiflora, with only 195 nucleotide sequences deposited in GenBank until 24 January 2014, which has restricted the development of modern breeding technologies.

Transcriptome sequencing (RNA-Seq) is a highly efficient and conventional molecular biology research method based on next-generation sequencing technology [6]. In recent years, the continuous development and improvement of this technology, combined with a more mature commercial operation, have provided new approaches and designs in the study of functional genomics of different plants and have made transcriptomics a new starting point and breakthrough point in plant research [7-9]. Compared with the gene chip technique, which is based on the premise of a large number of genes or molecular database information, RNA-Seq can be studied in non-model organisms without corresponding genome sequences as a reference [10-12]. Moreover, transcriptome study enables researchers to understand plant gene functions and structures at a specific stage and is more convenient to reveal the molecular mechanisms of specific biological processes [13]. Recently, RNA-Seq has become widely applied in many plants such as cucumber (Cucumis sativus L.) [14,15], Chinese cabbage (Brassica rapa L.) [16], blueberry (Vaccinium corymbosum L.) [17], peach (Prunus persica L. Batsch) [18], camelina (Camelina sativa L. Crantz) [19], etc. To the best of our knowledge, colour formation has been intensely investigated in fruits using RNA-Seq, and some information has been obtained regarding Unigenes, such as sweet orange (Citrus sinensis L. Osbeck) [20] and Chinese bayberry (Myrica rubra Sieb. and Zucc.) [21]. However, in flowers, RNA-Seq has been used in indepth studies of colour formation only in wintersweet (Chimonanthus praecox L.) [22] and safflower (Carthamus tinctorius L.) [23], and the flavonoid biosynthetic pathway has been annotated in these plants which is widespread in regulating flower colour formation of plants. In model plants, this biosynthetic pathway can be divided into three stages [24]. The first stage is the conversion of phenylalanine to coumarate-CoA by phenylalanine ammonialyase gene $(P A L)$, cinnamate 4-hydroxylase gene $(C 4 H)$ and 4-coumarate CoA ligase gene $(4 C L)$. The second stage is the formation of dihydroflavonol by one molecule of coumarate-CoA and three molecules of malonyl-CoA catalyzed by chalcone synthase gene $(\mathrm{CHS})$, chalcone isomerase gene $(C H I)$, flavanone 3-hydroxylase gene $(F 3 H)$, flavonoid 3 '-hydroxylase gene $\left(F 3^{\prime} H\right)$, flavonoid $3^{\prime}, 5^{\prime}$-hydroxylase gene $\left(F 3^{\prime} 5^{\prime} H\right)$ and flavonol synthase gene $(F L S)$. The third stage is the formation of various anthocyanidins by dihydroflavonols catalyzed by dihydroflavonol 4-reductase gene $(D F R)$ and anthocyanidin synthase gene (ANS). The synthesized anthocyanidins are then modified through a series of glycosylation and methylation steps to form stable anthocyanins catalyzed by anthocyanidin 3-O-glucosyltransferase gene (3GT), anthocyanidin 5-O-glucosyltransferase gene $(5 G T)$ and methyl transferase gene $(M T)$. However, there has been limited related information on P. lactiflora, and these studies provide very useful references for the study of yellow formation in P. lactiflora.

It is well known that plant materials with obvious differences are essential in the study of gene function. Furthermore, genetic mutants that control relative characteristics are the best materials to study gene function. In the present study, a flower colour chimaera cultivar 'Jinhui' 
with a consistent genetic background red outer-petal and yellow inner-petal was used as the experimental material including four developmental stages (S1, flowerbud stage; S2, initiating bloom; S3, bloom stage; and S4, wither stage), and Illumina sequencing was adopted to analyse their difference of transcriptome. Based on this, the expression patterns of differentially expressed genes (DEGs) identified in the flavonoid biosynthetic pathway were analysed using real-time quantitative polymerase chain reaction (Q-PCR), and qualitative and quantitative analysis of flavonoids was performed using highperformance liquid chromatograph (HPLC)-electrospray ionization (ESI)-mass spectrometry $\left(\mathrm{MS}^{\mathrm{n}}\right)$ technology, thereby revealing the biochemical and molecular mechanisms of yellow formation in P. lactiflora. Taken together, these results would provide a foundation for breeding yellow P. lactiflora varieties.

\section{Results}

\section{Colour indices}

To obtain the same genetic background, a flower colour chimaera cultivar 'Jinhui' with red outer-petal and yellow inner-petal was used to study the mechanism of yellow formation in P. lactiflora (Figure 1). Firstly, their colour indices were measured. On one hand, the flower colours were defined according to the Royal Horticultural Society Color Chart (RHSCC). During flower development, the colour gradation of the outer-petal ranged from $164 \mathrm{~A}, 73 \mathrm{~A}$ and $75 \mathrm{~B}$ to $75 \mathrm{C}$, while that of the inner-petal changed from $11 \mathrm{~A}, 11 \mathrm{~B}$ and $11 \mathrm{C}$ to $11 \mathrm{D}$. On the other hand, flower colours were expressed as $L^{\prime \prime}, a^{\prime \prime}$ and $b^{\prime \prime}$. In a uniform colour space, $L^{*}$ represented lightness, $a^{\prime \prime}$ represented the ratio of red/magenta and green, and $b^{*}$ represented the ratio of yellow and blue [25]. In four different developmental stages, $L^{*}$ values of the outer-petal and inner-petal were all gradually increased which was always lower in the outer-petal compared to the inner-petal; however, the $b^{*}$ value of the outer-petal and $a^{*}$ value of the inner-petal decreased, which all tended to zero. These colour indices showed that the colours of the outer-petal and inner-petal were pure red and yellow in S1, respectively, and then faded gradually with flower development. In addition, the flower diameter was also measured, such that the whole flower and inner-petal were similar and had constantly increased from S1 to S4. Taken together, these measured results were consistent with the visual results.

\section{RNA-Seq and de novo assembly}

To deeply elucidate the molecular mechanism underlying yellow colour formation, RNA-Seq analysis were performed using the Illumina platform. Each library of the outer-petal and inner-petal produced 66,179,398 and $65,481,444$ total raw reads, following cleaning and quality checks, and 60,234,256 and 61,296,620 total clean reads were obtained with a single read length of $90 \mathrm{nt}$ and a Q20 percentage (proportion of nucleotides with quality value larger than 20 in reads) over $98.50 \%$ (Table 1). These data showed that the RNA-Seq quality was better, which can be used for further analysis.

Subsequently, short reads from the outer-petal and inner-petal were assembled into 107,199 and 127,518 Contigs, which contained an average length of 360 and $337 \mathrm{nt}$, together with N50 length (covering 50\% all the nucleotide sequences of the largest Contig length) of 745 and $664 \mathrm{nt}$, respectively. After linking the Contigs together, 61,431 and 70,359 Unigenes with an average length of 628 and $617 \mathrm{nt}$ of the outer-petal and innerpetal were identified, respectively, and their N50 values were all 1047 nt. Finally, 61,408 non-redundant Allunigenes were obtained according to splicing and reducing the redundancy of the Unigenes sequence in the outerpetal and inner-petal, and its average length and N50 value were $763 \mathrm{nt}$ and $1219 \mathrm{nt}$, respectively (Table 2). Among these, there were 32,517 Unigenes with a length of 100 to $500 \mathrm{nt}$, which accounted for $52.92 \%$, and excluding $\mathrm{N}$ rate reached $100 \%$. All these results showed that the assembly quality was sufficiently high for gene function analysis. In addition, the distributions of the reads in All-unigenes were analysed, and these results showed that the number of reads in the $3^{\prime}$ and $5^{\prime}$ ends from All-unigenes was relatively low, particularly in the $3^{\prime}$ and $5^{\prime}$ tail ends. However, the number of reads located in 0.2 to 0.8 was relatively high, and its distribution was balanced (Additional file 1: Figure S1).

\section{Functional annotation}

Due to the lack of a complete genome sequence in P. lactiflora, 61,408 All-unigenes were blasted against six public databases, i.e., non-redundant protein database (NR), Swiss-Prot protein database (Swiss-Prot), Kyoto encyclopedia of genes and genomes database (KEGG), cluster of orthologous groups of proteins database (COG), gene ontology database (GO) and non-redundant nucleotide database (NT). These results revealed that 37,511 Allunigenes were annotated, which accounted for $61.08 \%$. Of the All-unigenes, 35,972, 30,199 and 26,674 All-unigenes could be annotated to NR, NT and GO databases, accounting for $58.57 \%, 49.17 \%$ and $43.43 \%$, respectively, whereas in the Swiss-Prot, KEGG and COG databases, only 22,655, 20,294 and 13,089 All-unigenes were annotated, which accounted for $36.89 \%, 33.04 \%$ and $21.31 \%$. For the NR annotation, the ratio of the All-unigenes distributed in $10^{-15}<\mathrm{e}$-value $<10^{-5}$ and $0<\mathrm{e}$-value $<10^{-100}$ with $17.4 \%$ and $16.9 \%$ was maximum; $67.4 \%$ of Allunigenes showed $60-95 \%$ similarity; and 30,627 Allunigene sequences shared a similarity with available plant sequences from Vitis vinifera, P. persica, Ricinus 


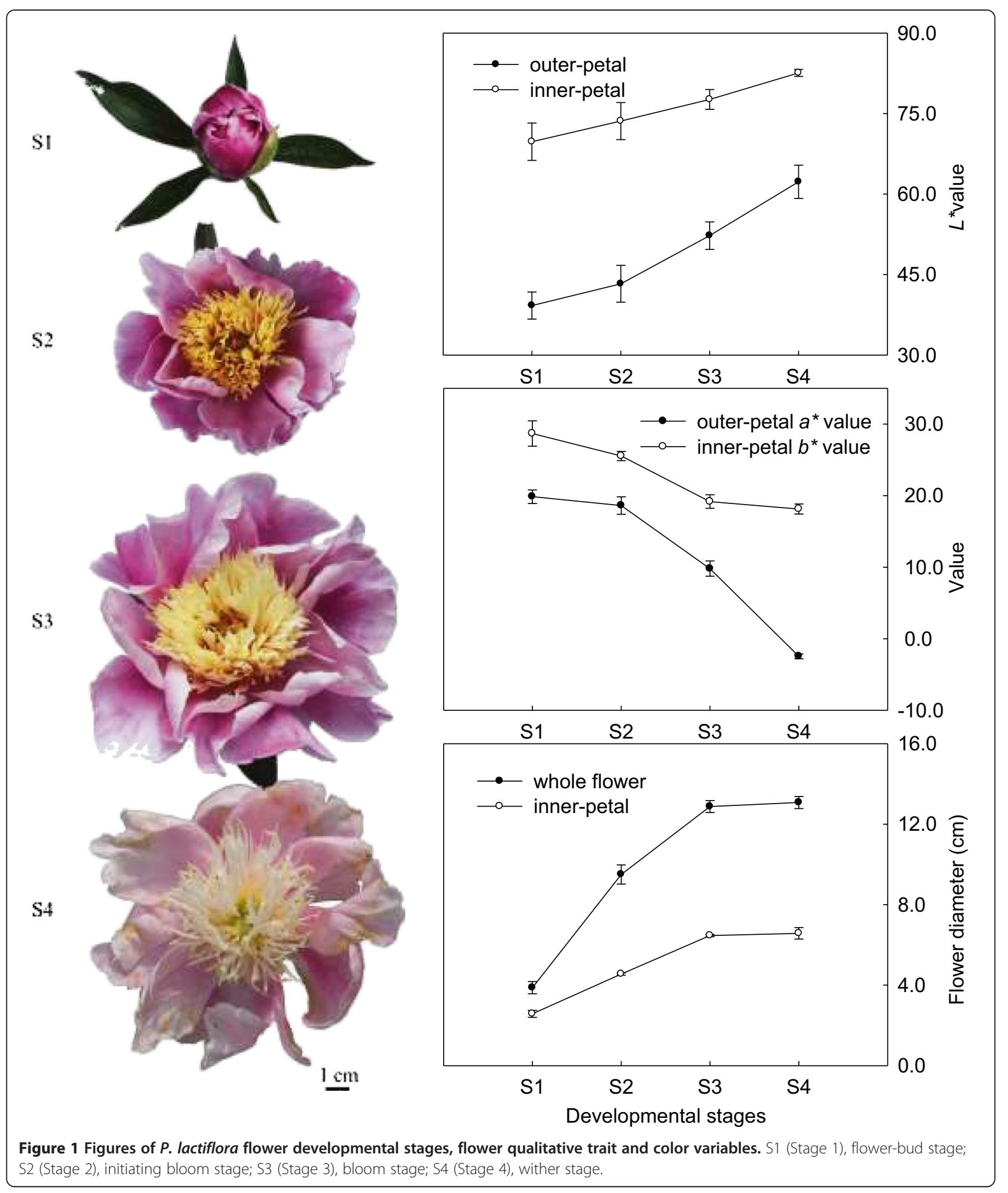

communis, Populus trichocarpa, Fragaria vesca subsp. Vesca, C. sativus and Glycine max, including up to 48.5\% similarity with Vitis vinifera (Additional file 2: Figure S2).
When compared with the COG database, 13,089 Allunigenes were divided into 25 different functional classifications, and the functional types comprehensively involved most of the life activities (Figure 2). Among 
Table 1 Output statistics of RNA-Seq of $P$. lactiflora outer-petal and inner-petal

\begin{tabular}{ccccccc}
\hline Samples & Total raw reads & Total clean reads reads & Total clean nucleotides & Q20 percentage & N percentage & GC percentage \\
\hline outer-petal & $66,179,398$ & $60,234,256$ & $5,421,083,040$ & $98.52 \%$ & $0.00 \%$ & $45.76 \%$ \\
inner-petal & $65,481,444$ & $61,296,620$ & $5,516,695,800$ & $98.58 \%$ & $0.00 \%$ & $45.22 \%$ \\
\hline
\end{tabular}

these types, the maximum number of All-unigenes was categorised as general function prediction only $(4,158$ All-unigenes, $16.71 \%)$, followed by transcription $(2,284$ All-unigenes, 9.18\%), replication, recombination and repair (2,067 All-unigenes, 8.31\%) and posttranslational modification, protein turnover, chaperones (1,917 Allunigenes, $7.70 \%$ ), while the minimum number was classified to nuclear structure (2 All-unigenes, 0.008\%).

\section{Comparative analysis of DEGs and GO functional} annotation between the outer-petal and inner-petal

To identify key genes controlling yellow formation in P. lactiflora, the transcriptomes of the outer-petal and inner-petal were comparatively analysed based on the outer-petal as the control group. As shown in Figure 3, 6,345 DEGs were obtained, among which, the expression levels of 3,899 DEGs (red scatters) were up-regulated and the expression levels of 2,446 DEGs (green scatters) were down-regulated. Furthermore, abundant blue scatters indicated no difference in expression between the outer-petal and inner-petal. On the basis of sequence homology, these 6,345 DEGs could be classified into three categories in GO assignments: biological process, cellular component and molecular function, and they embraced 22, 15 and 13 functional classifications, respectively (Figure 4). In the biological process category, cellular process $(1,786$ DEGs, $15.74 \%)$ and metabolic process (1,758 DEGs, $15.49 \%)$ accounted for the major proportion. In the cellular component category, 1,894 and 1,894 DEGs (23.37\%) were enriched in the cell and cell part. Under the molecular function category, catalytic activity $(1,649$ DEGs, 44.41\%) and binding (1,412 DEGs, 38.03\%) were dominant functions.

\section{Functional classification using KEGG}

The pathway database was the major component of KEGG, which contained most of the metabolic pathways representing information on the molecular interaction and reaction networks [26]. In this study, 6,345 DEGs were subsequently analysed in the KEGG pathway database, where 2,559 DEGs (40.33\%) were assigned to 121 KEGG pathways and only 37 pathways met the condition of Q-value $\leq 0.05$ (Figure 5, Additional file 3: Table S1). Among these pathways, metabolic pathways demonstrated the largest number of DEGs (796 DEGs, $31.11 \%$, ko01100), followed by biosynthesis of secondary metabolites (429 DEGs, 16.76\%, ko01110), plant-pathogen interaction (189 DEGs, 7.39\%, ko04626), plant hormone signal transduction (156 DEGs, 6.1\%, ko04075), endocytosis (146 DEGs, 5.71\%, ko04144), starch and sucrose metabolism (145 DEGs, 5.67\%, ko00500) and glycerophospholipid metabolism (142 DEGs, 5.55\%, ko00564). Many studies revealed that colour development of the P. lactiflora petal was closely related to flavonoids [27-29], and our previous study also confirmed this conclusion [30]. As expected, five pathways related to colour development were identified, namely phenylpropanoid biosynthesis (82 DEGs, 3.2\%, ko00940), flavonoid biosynthesis (43 DEGs, 1.68\%, ko00941), flavone and flavonol biosynthesis (39 DEGs, 1.52\%, ko00944), isoflavonoid biosynthesis (16 DEGs, 0.63\%, ko00943) and anthocyanin biosynthesis (8 DEGs, 0.31\%, ko00942), and they all belonged to the flavonoid metabolic pathway which could be establish connection taking flavonoid biosynthesis as the ligament.

\section{Expression analysis of candidate DEGs related to colour development}

According to the genes annotated in KEGG and the results of flower colour formation obtained in many plants [31,32], 10 DEGs were selected for expression analysis (Table 3). Among these DEGs, PAL, CHI, F3H, F3' $H$, $D F R, A N S$, 3GT and 5GT had been registered in GenBank, and their accession numbers were JQ070801, JN119872, JQ070802, JQ070803, JQ070804, JQ070805, JQ070806 and JQ070807, respectively. However, F3'5' $H$ and FLS were

Table 2 Data assembly of transcriptome Contig and Unigene in P. lactiflora outer-petal and inner-petal

\begin{tabular}{|c|c|c|c|c|c|c|c|c|}
\hline & Sample & Total number & Total length (nt) & Mean length (nt) & N50 & $\begin{array}{c}\text { Total consensus } \\
\text { sequences }\end{array}$ & $\begin{array}{l}\text { Distinct } \\
\text { clusters }\end{array}$ & $\begin{array}{c}\text { Distinct } \\
\text { singletons }\end{array}$ \\
\hline \multirow[t]{2}{*}{ Contig } & outer-petal & 107,199 & $38,543,748$ & 360 & 745 & - & - & - \\
\hline & inner-petal & 127,518 & $42,986,411$ & 337 & 664 & - & - & - \\
\hline \multirow[t]{3}{*}{ Unigene } & outer-petal & 61,431 & $38,554,638$ & 628 & 1047 & 61,431 & 16,143 & 45,288 \\
\hline & inner-petal & 70,359 & $43,390,011$ & 617 & 1047 & 70,359 & 20,147 & 50,212 \\
\hline & All & 61,408 & $46,873,884$ & 763 & 1219 & 61,408 & 20,191 & 41,217 \\
\hline
\end{tabular}




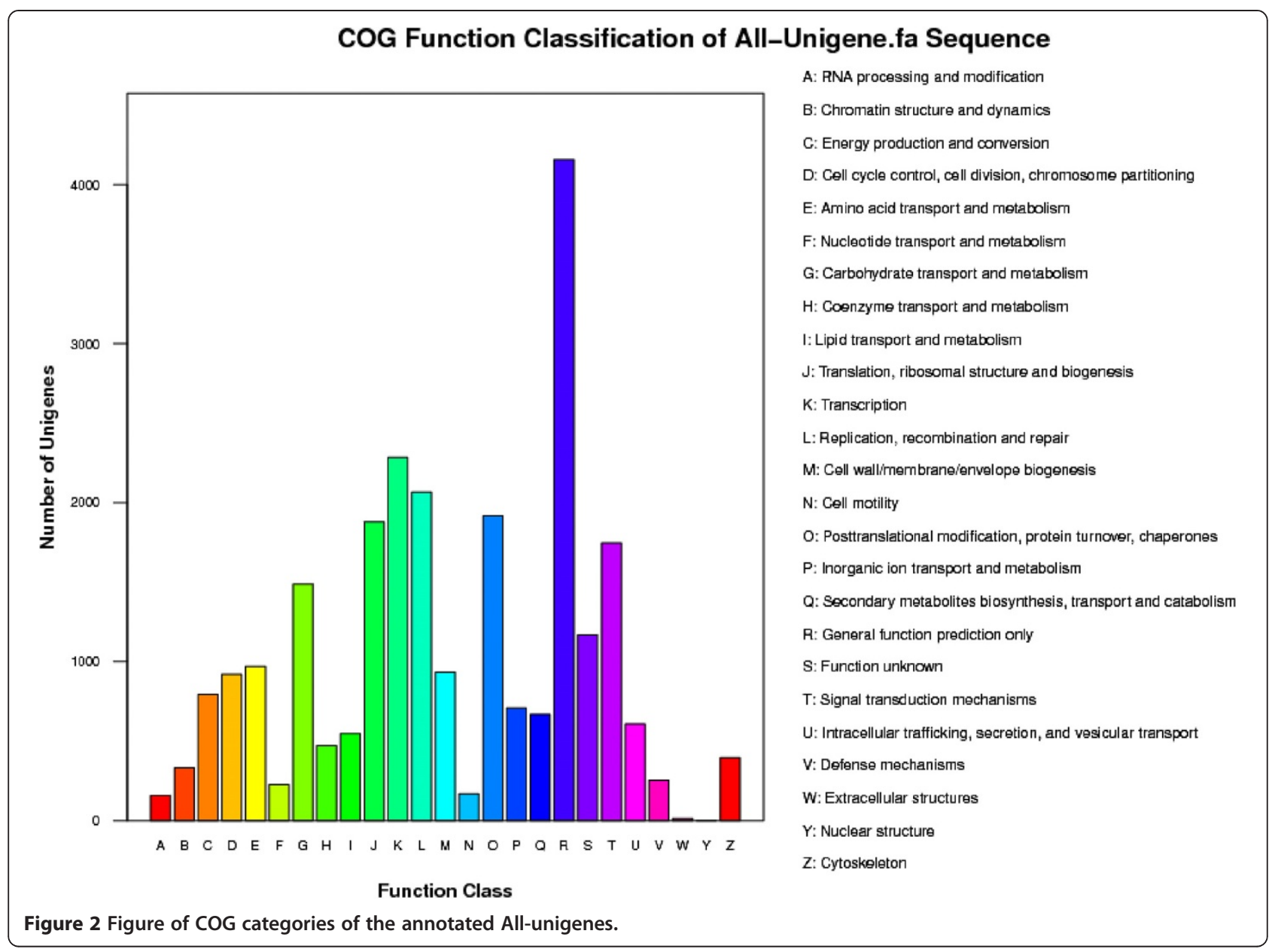

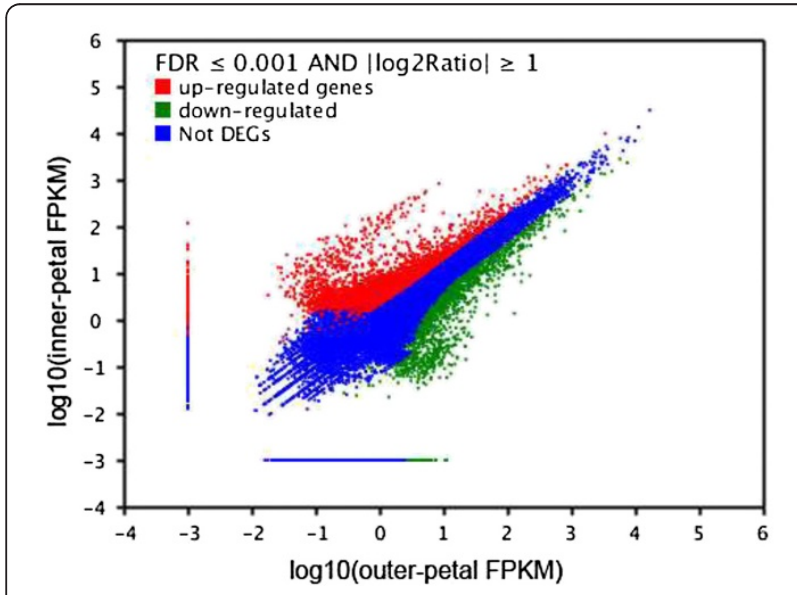

Figure 3 Figure of DEGs in P. lactiflora outer-petal and inner-petal. Red scatters indicate up-regulated DEGs, green scatters indicate down-regulated DEGs, and blue scatters indicate no difference DEGs in expression between the outer-petal and inner-petal. first found in P. lactiflora. Among them, more than one Unigene was annotated as the same enzyme, which might be because these Unigenes represented different members of the same gene family, different fragments of a single transcript, or both, except $P A L$ and $F 3^{\prime} 5^{\prime} H$ [33]. As shown in Figure 6, Q-PCR was used to study their expression patterns in four different developmental stages of the outerpetal and inner-petal. Single gene expression level analyses indicated that although the expressions of all 10 DEGs were detected during flower development, but their expression levels were different. Among the 10 DEGs, the expression levels of $P l F 3 H$ and $P l F 3^{\prime} H$ were the highest, and downstream PlDFR and Pl5GT exhibited the lowest expression levels, while the other DEGs ranged between the two genes listed above. In terms of the overall trend, the expression levels of the 10 DEGs were similar during the outer-petal and inner-petal development except in some stages. For example, the expression levels of PlFLS and Pl3GT increased until they reached the maximum level during flower development, the other 8 DEGs all showed a downward trend, and they only had some differences in quantity between the outer-petal and inner-petal. 

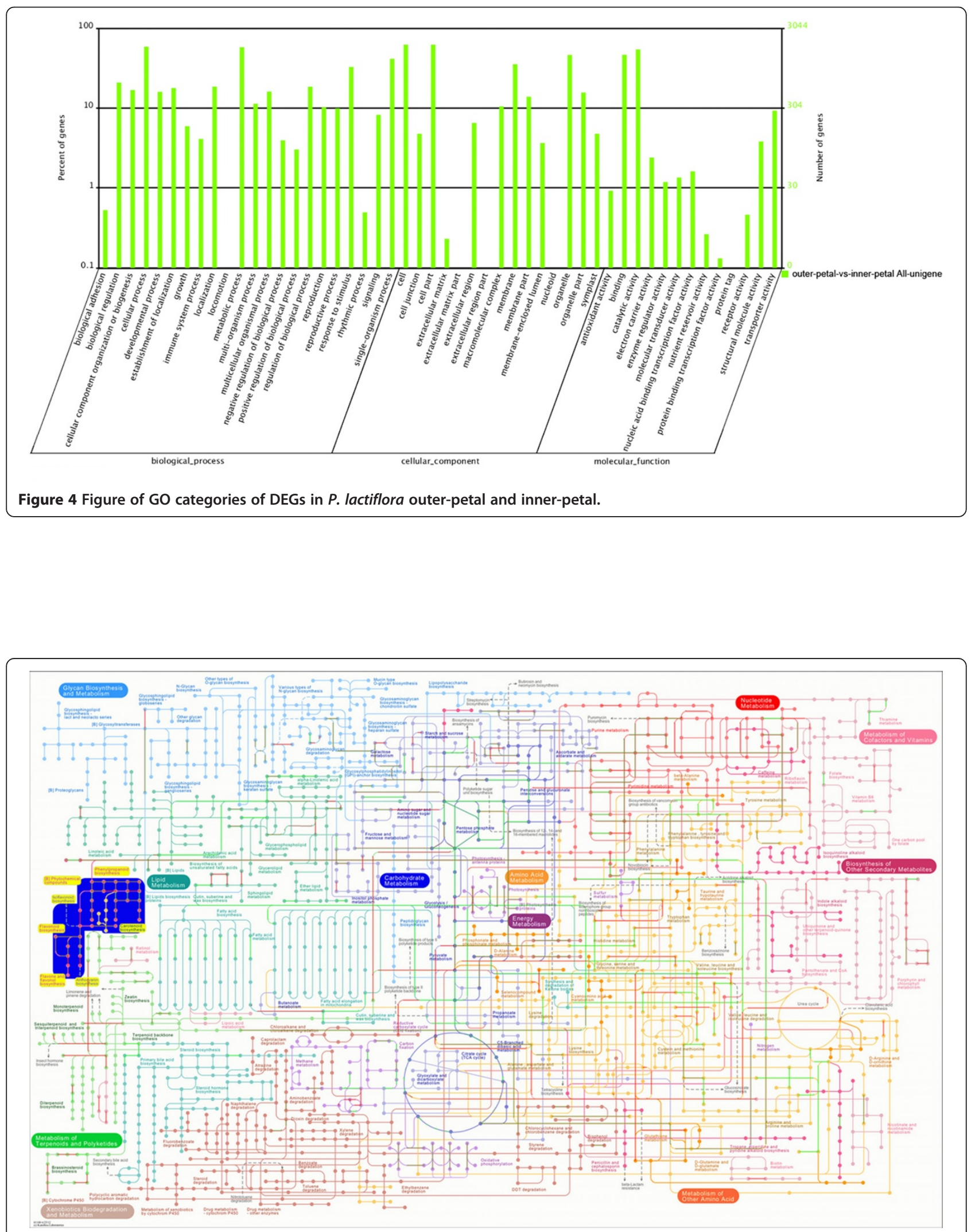

Figure 5 Interactive pathways during the outer-petal and inner-petal development. Blue part is flavonoid metabolic pathway related to colour development. 
Table 3 Candidate DEGs involved in P. lactiflora colour development

\begin{tabular}{|c|c|c|c|c|c|c|}
\hline Gene & Enzyme & Enzyme no. & KO id & No. all & No. up & No. down \\
\hline PAL & Phenylalanine ammonia-lyase & [EC: 4.3.1.24] & K10775 & 1 & 0 & 1 \\
\hline $\mathrm{CHI}$ & Chalcone isomerase & [EC: 5.5 .1 .6$]$ & K01859 & 2 & 2 & 0 \\
\hline $\mathrm{F} 3 \mathrm{H}$ & Flavanone 3-hydroxylase & [EC: 1.14.11.9] & K00475 & 2 & 1 & 1 \\
\hline$F 3^{\prime} H$ & Flavonoid 3'-hydroxylase & [EC: 1.14.13.21] & K05280 & 14 & 5 & 9 \\
\hline $\mathrm{F}^{\prime} 5^{\prime} \mathrm{H}$ & Flavonoid 3',5'-hydroxylase & [EC: 1.14.13.88] & K13083 & 1 & 1 & 0 \\
\hline$F L S$ & Flavonol synthase & [EC: 1.14.11.23] & K05278 & 6 & 1 & 5 \\
\hline$D F R$ & Dihydroflavonol 4-reductase & [EC: 1.1.1.219] & K13082 & 3 & 2 & 1 \\
\hline ANS & Anthocyanidin synthase & [EC: 1.14.11.19] & K05277 & 4 & 0 & 4 \\
\hline $3 G T$ & Anthocyanidin 3-O-glucosyltransferase & [EC: 2.4.1.115] & K12930 & 3 & 3 & 0 \\
\hline $5 G T$ & Anthocyanidin 5-O-glucosyltransferase & [EC: 2.4.1.298] & K12338 & 5 & 1 & 4 \\
\hline
\end{tabular}

No. All indicates the total number of DEGs, No. Up indicates the number of DEGs in the inner-petal compared with the outer-petal in S2, No. Down indicates the number of DEGs in the inner-petal compared with the outer-petal in S2.

For different petals, the first key gene in flavonoid metabolism PlPAL; PIDFR controlling the formation of leucoanthocyanidin; and Pl3GT together with Pl5GT, which catalyses the formation of the complex aglycone of anthocyanin composition, all had higher expression levels in the outer-petal compared to the inner-petal in the four different developmental stages, whereas $F 3^{\prime} 5^{\prime} H$ showed an opposite trend.

\section{Qualitative and quantitative analysis of flavonoids}

To examine whether flavonoid accumulation in P. lactiflora petals was consistent with the expression patterns of candidate DEGs related to colour development, qualitative and quantitative analysis of flavonoids were performed using HPLC-ESI-MS ${ }^{\mathrm{n}}$ in the same samples used in the expression analysis. On the basis of the ultraviolet-visible absorption characteristics, anthocyanins, anthoxanthins and chalcones were detected under the wavelength of $525 \mathrm{~nm}, 350 \mathrm{~nm}$ and $365 \mathrm{~nm}$, respectively. As shown in Figure 7, the chromatographic peaks of the outer-petal and inner-petal were identical, although there were only some differences in the peak area. The HPLC retention time and ultraviolet-visible spectral properties together with mass spectrometric data of identified main chromatographic peaks were listed in Additional file 4: Table S2. Moreover, two (a1 and a2) and seven $(\mathrm{f} 1, \mathrm{f} 2, \mathrm{f} 3, \mathrm{f} 4, \mathrm{f} 5, \mathrm{f} 6$ and $\mathrm{f} 7)$ peaks were identified at $525 \mathrm{~nm}$ and $350 \mathrm{~nm}$, namely cyanidin-3,5-di-O-glucoside, peonidin-3,5-di-O-glucoside, kaempferol di-hexoside, kaempferol-3-O-malonylglucoside-7-O-glucoside, quercetin3-O-galactoside, luteolin-7-O-galloylglucoside, luteolin7-O-glucoside, isorhamnetin-3-O-glucoside and flavone $\mathrm{C}$-glycoside, respectively.

Relevant standards were used as the references for the relative quantitative analysis of flavonoids, the results showed that there were tremendous differences in both the two different petals and also during different developmental stages of the same petals (Table 4). For the composition, peonidin-3,5-di-O-glucoside was the main component of anthocyanins in P. lactiflora outerpetal and inner-petal, which accounted for approximately $73.20 \%$ and $65.94 \%$ of the total anthocyanin contents in the outer-petal and inner-petal, respectively. In addition, the main component of anthoxanthins in P. lactiflora outer-petal and inner-petal was luteolin-7-O-glucoside, which comprised approximately $33.69 \%$ and $36.04 \%$ of the total anthoxanthin contents in the outer-petal and inner-petal, respectively, which was followed by kaempferol di-hexoside (17.45\% and 19.41\%), luteolin-7$O$-galloylglucoside (16.14\% and $9.21 \%)$ and flavone Cglycoside (9.99\% and $10.93 \%)$. Concerning the content of composition, the results showed that the content of each composition in the outer-petal was basically higher compared to the inner-petal, particularly for a1 and a2 of anthocyanins. In $\mathrm{S} 1$, the contents of a1 and a2 in the outer-petal were 11 times as much as that of the innerpetal and increased to 30 times in S4. In the case of different developmental stages, each anthocyanin and anthoxanthin composition in the outer-petal were basically decreased from S1 to S3, and slightly increased in S4. However, the compositions in the inner-petal were always decreased with flower development, except f1, f4 and $\mathrm{f7}$ in S4. For total content, the total contents of anthocyanins, anthoxanthins and flavonoids in the outerpetal were all higher compared to the inner-petal, and specifically, the difference in anthocyanins was achieved by a multiple of 10 . Meanwhile, the total contents of anthocyanins, anthoxanthins and flavonoids in the outerpetal and inner-petal all presented a downward trend as flower development, although there was an increase in the outer-petal at S4. Furthermore, the co-pigment index (CI), which determined whether had the co-pigment effect between anthoxanthins and anthocyanins demonstrated an increasing tendency in both the outer-petal and 

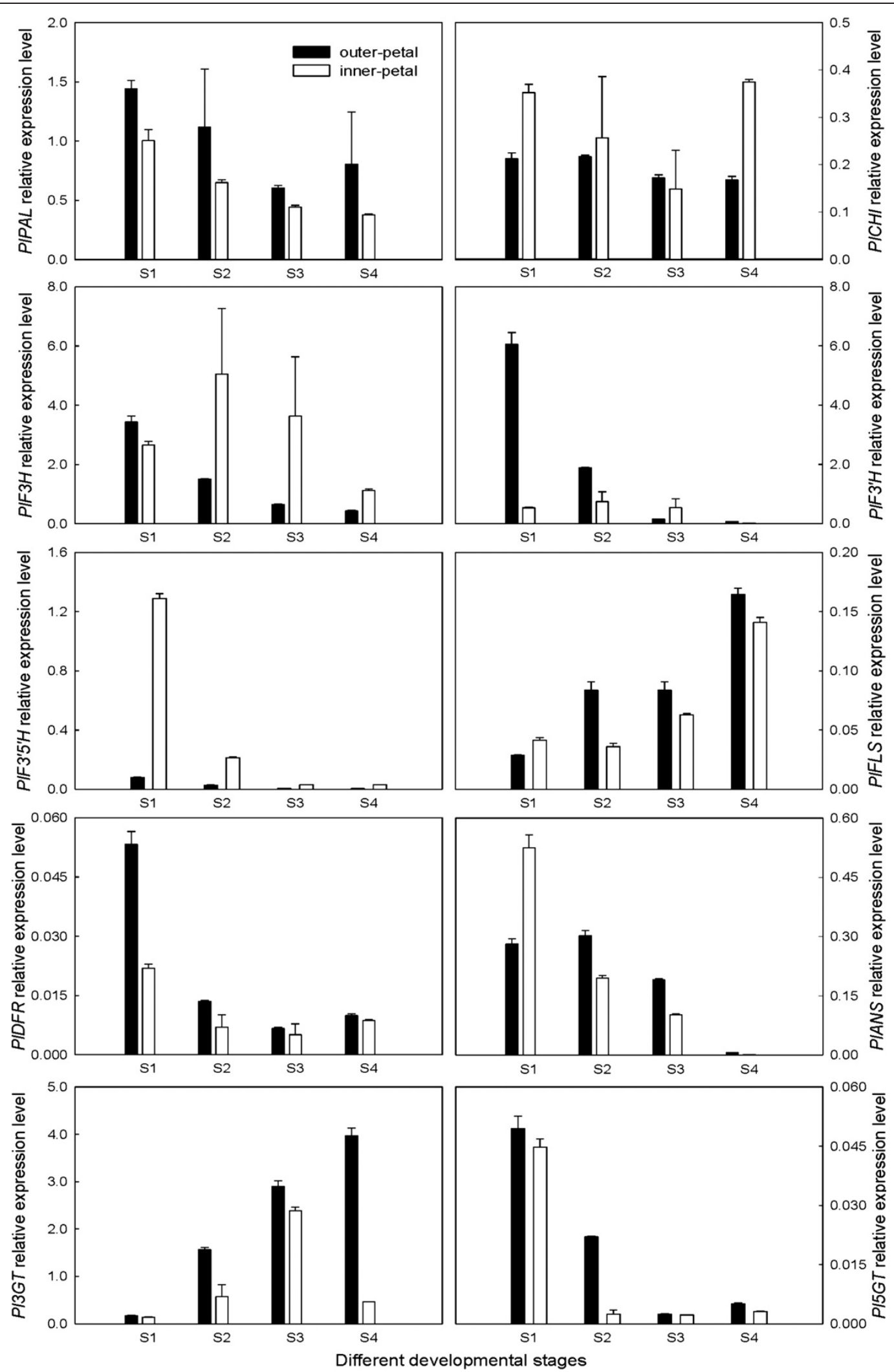

Figure 6 Expression analysis of 10 candidate DEGs related to colour development in P. lactiflora. 

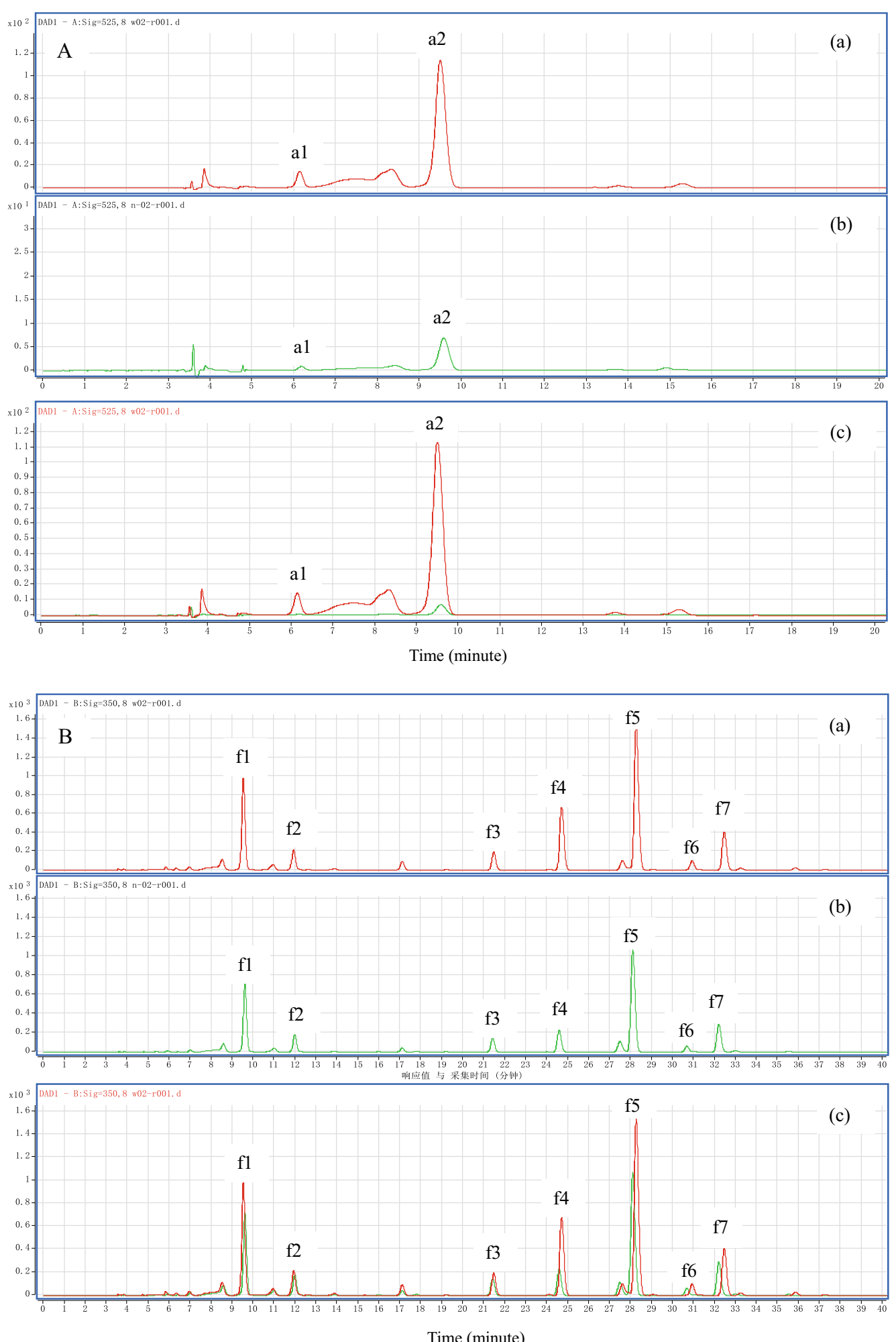

Figure 7 HPLC chromatograms of $P$. lactiflora anthocyanins (A, detected at $525 \mathrm{~nm}$ ) and anthoxanthins (B, detected at $350 \mathrm{~nm}$ ) in S2.

(a) indicates HPLC chromatograms of outer-petal; (b) indicates HPLC chromatograms of inner-petal; (c) indicates HPLC chromatograms of (a) and (b) merge together. a1-a2 indicates identified anthocyanins; f1-f7 indicates identified anthoxanthins. 
Table 4 Contents of anthocyanin and anthoxanthin compositions in P. lactiflora petals during flower development (ug g (1) $^{-1}$ )

\begin{tabular}{|c|c|c|c|c|c|c|c|c|}
\hline \multirow{2}{*}{ Peak } & \multicolumn{2}{|c|}{ S1 } & \multicolumn{2}{|c|}{ S2 } & \multicolumn{2}{|c|}{ S3 } & \multicolumn{2}{|c|}{ S4 } \\
\hline & outer-patal & inner-petal & outer-patal & inner-petal & outer-patal & inner-petal & outer-patal & inner-petal \\
\hline a1 & $149.78 \pm 0.96$ & $12.46 \pm 0.21$ & $134.69 \pm 0.90$ & $7.40 \pm 0.16$ & $98.90 \pm 1.92$ & $5.72 \pm 0.18$ & $135.37 \pm 1.28$ & $3.64 \pm 0.06$ \\
\hline a2 & $1979.83 \pm 0.34$ & $171.44 \pm 0.06$ & $1719.72 \pm 10.97$ & $103.33 \pm 0.25$ & $1210.93 \pm 26.70$ & $84.84 \pm 1.60$ & $1490.26 \pm 12.52$ & $49.10 \pm 0.71$ \\
\hline f1 & $2202.62 \pm 0.37$ & $1517.01 \pm 2.59$ & $1812.72 \pm 9.11$ & $1317.25 \pm 3.11$ & $1280.69 \pm 21.37$ & $1198.32 \pm 12.98$ & $1991.88 \pm 14.29$ & $1208.84 \pm 12.56$ \\
\hline f2 & $560.75 \pm 1.57$ & $470.36 \pm 0.73$ & $459.87 \pm 0.51$ & $371.93 \pm 1.14$ & $359.03 \pm 4.97$ & $318.48 \pm 2.51$ & $497.65 \pm 2.17$ & $314.18 \pm 2.74$ \\
\hline f3 & $448.96 \pm 0.29$ & $421.65 \pm 7.23$ & $436.23 \pm 0.98$ & $311.22 \pm 9.74$ & $365.77 \pm 3.66$ & $263.00 \pm 1.73$ & $483.65 \pm 1.93$ & $191.72 \pm 0.30$ \\
\hline $\mathrm{f} 4$ & $1834.72 \pm 14.07$ & $558.33 \pm 1.48$ & $1599.16 \pm 9.53$ & $532.38 \pm 2.31$ & $1339.25 \pm 8.09$ & $599.89 \pm 3.60$ & $1968.99 \pm 16.64$ & $795.47 \pm 3.37$ \\
\hline f5 & $4303.42 \pm 15.01$ & $3088.60 \pm 1.24$ & $3609.96 \pm 9.52$ & $2494.02 \pm 7.83$ & $2607.99 \pm 1.78$ & $2181.54 \pm 1.34$ & $3554.77 \pm 6.46$ & $1968.36 \pm 1.42$ \\
\hline f6 & $244.87 \pm 4.51$ & $186.11 \pm 2.51$ & $225.65 \pm 2.33$ & $136.93 \pm 0.73$ & $187.03 \pm 0.85$ & $112.52 \pm 1.28$ & $255.83 \pm 1.59$ & $87.42 \pm 1.37$ \\
\hline f7 & $1175.88 \pm 2.61$ & $948.82 \pm 9.70$ & $1016.03 \pm 3.68$ & $718.43 \pm 2.48$ & $870.68 \pm 16.73$ & $627.85 \pm 2.05$ & $1110.19 \pm 8.76$ & $657.09 \pm 19.84$ \\
\hline
\end{tabular}

S1 (Stage 1), flower-bud stage; S2 (Stage 2), initiating bloom stage; S3 (Stage 3), bloom stage; S4 (Stage 4), wither stage.

inner-petal. In the outer-petal, the CI had increased from 4.55 in S1 to 5.51 in S4, in contrast, and its developmental difference in the inner-petal was more significant and increased from 34.00 in S1 to 69.28 in S4 (Figure 8).

\section{Discussion}

$P$. lactiflora is an important ornamental plant with a cultivation history of more than 4000 years in China. Due to abundant flower colours, variable flower shapes, sharp flower fragrances and attractive flower poses, $P$. lactiflora exhibits a very high ornamental value and rich landscaping application value, which has become increasingly favoured by people worldwide. However, compared with plants in which genome sequencing has been completed, such as arabidopsis (Arabidopsis thaliana (L.) Heynh.) [34], mei (Prunus mume Sieb. et Zucc.) [35], lotus (Nelumbo nucifera Gaertn.) [36], spider flower (Tarenaya hassleriana (Chodat) Iltis) [37] and carnation (Dianthus caryophyllus L.) [38], a weak molecular basis and an incompletely clear genetic background in P. lactiflora severely limit its in-depth study. Transcriptome refers to the identity of each expressed gene and its expression level for cells in a particular state, whose expression largely

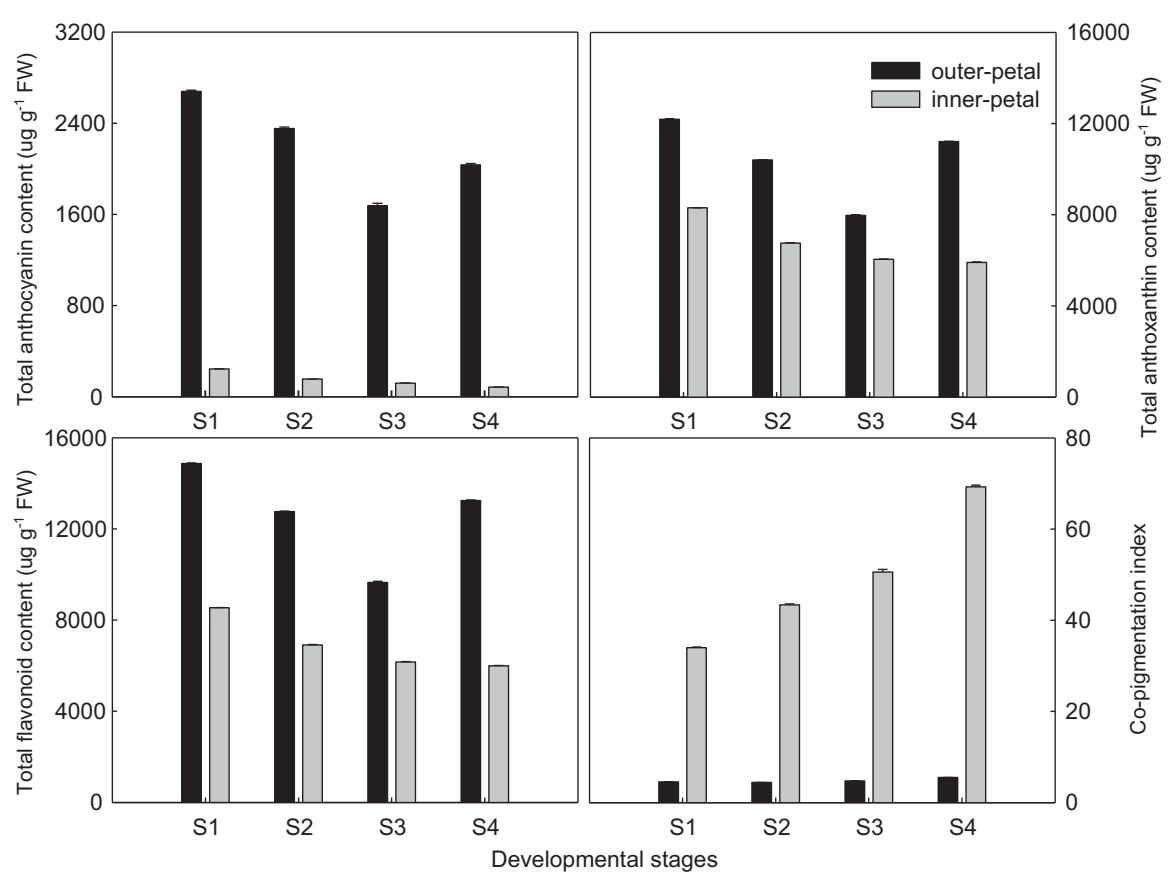

Figure 8 Total content of anthocyanins, anthoxanthins and flavonoids, and co-pigmentation index in P. lactiflora outer-petal and inner-petal during flower development. 
controls the phenotype of an organism. Unlike the genome, the transcriptome is more dynamic and provides more valuable reference information for cell function interpretation, but its study is more inexpensive [39]. Due to overcoming technological limitations, transcriptome studies have been extensively developed in plants [32,40-42]. Moreover, according to a different purpose of the study, RNA-Seq can be divided into two methods: on one hand, different experimental materials are mixed equally to sequence, which can obtain the largest number of unique transcripts [21,23]; on the other hand, different experimental materials are sequenced separately, which can isolate tissue-specific expression genes $[21,43,44]$. Wang et al. aimed to elucidate the transcriptome profiling of radish (Raphanus sativus L.) root and identify the critical gene response to lead $(\mathrm{Pb})$ stress, two radish root cDNA samples, where one untreated control and the other $\mathrm{Pb}$-stressed with $\mathrm{Pb}\left(\mathrm{NO}_{3}\right)_{2}$ at $1000 \mathrm{mg}$ were separately sequenced, and 4,614 DEGs were obtained [45]. In this study, to characterize the transcriptome of $P$. lactiflora red outer-petal and yellow inner-petal and identify the critical genes mediating yellow formation, Illumina sequencing was firstly employed to the outerpetal and inner-petal with significantly different colours separately. Each library of the outer-petal and inner-petal produced 5,421,083,040 and 5,516,695,800 total clean nucleotides with a Q20 percentage over $98.50 \%$ in which the quantity and quality were all relatively high compared with other plants, such as the apple tree [46] and M. rubra [21]. Subsequently, 61,431 and 70,359 unigenes with an average length of 628 and $617 \mathrm{nt}$ were found in the outerpetal and inner-petal, and 6,345 DEGs were identified, which provided a large amount of information for molecular biological research in P. lactiflora.

Colour formation is a complex process that includes many metabolic pathways and gene expression. In the transcriptome study of colour, $\mathrm{Xu}$ et al. speculated that enhanced photosynthesis and reduced downstream gene expression in the carotenoid biosynthetic pathway were the key reason for sweet orange red-flesh mutant lycopene accumulation, which controlled red formation [47]. Recently, two varieties of flesh in four developmental stages were used to perform RNA-Seq by this research group, which further confirmed the correlation between carotenoid metabolism and red colour formation in sweet orange red-flesh mutant [20]. In M. rubra, Feng et al. aimed to obtain a general overview of the transcriptome, four libraries containing mixed tissues, and three different developmental fruits were designed for RNA-Seq [21]. This study identified 41,239 Unigenes, generated interactive pathways during bayberry fruit ripening, and found that the expression levels of flavonoid biosynthetic genes were overall increased, which was consistent with flavonoid accumulation and more intense colour. To the best of our knowledge in flower colour, transcriptome studies had been performed only on C. praecox [42] and $C$. tinctorius [23]. In the transcriptome database of C. praecox, 31,142 Unigenes could be annotated in 266 different metabolic pathways. Furthermore, 105 Unigenes with an average length of $677 \mathrm{bp}$ were associated with the metabolic pathway of flower colour, including the flavonoid biosynthetic pathway (70 Unigenes), flavone and flavonol biosynthetic pathway (24 Unigenes) and anthocyanin biosynthetic pathway (11 Unigenes), which was convenient for gene cloning and function identification [42]. Otherwise, the flavonoid biosynthetic pathway (274 Unigenes), flavone and flavonol biosynthetic pathway (114 Unigenes) and anthocyanin biosynthetic pathway (20 Unigenes) were identified according to the RNA-Seq comparison of red and white C. tinctorius. Moreover, the expression levels of 12 flavonoid biosynthetic pathway-related candidate Unigenes were analysed, indicating that CHS (Unigene96945) was very important for C. tinctorius flavonoid synthesis [23]. In the present study, 2,559 DEGs in the outer-petal and inner-petal had been annotated to 121 metabolic pathways, and there were 37 metabolic pathways enriched DEGs, which revealed the formation process complexity of different petals. Among these pathways, the flavonoid metabolic pathway containing phenylpropanoid biosynthesis, flavonoid biosynthesis, anthocyanin biosynthesis, isoflavonoid biosynthesis, flavone and flavonol biosynthesis was annotated, which validated the conclusion that flavonoids were the pigment of P. lactiflora petal $[27,28,30]$. Moreover, carotenoid biosynthesis and zeatin biosynthesis were also found in metabolic pathways, which might be resulted from incomplete petalody stamens. Interestingly, 7 glucide metabolic pathways, including pentose and glucuronate interconversions, starch and sucrose metabolism, glycolysis/gluconeogenesis, other glycan degradation, fructose and mannose metabolism, amino sugar and nucleotide sugar metabolism and galactose metabolism were annotated. These pathways were not only associated with the growth and development of petals but were also related to the synthetic substrates of anthocyanins.

Flavonoid biosynthesis, which was controlled by many genes [30], and key structural genes in the flavonoid metabolic pathway were screened in this study. Interestingly, the well-known key chalcone synthase gene $(\mathrm{CHS})$ catalyzing the formation of the chalcone derivative could not be found, which was the committed step leading to the biosynthesis of flavonoids, isoflavonoids and anthocyanins [48]. This might be the reason that the expression of PlCHS in the inner-petal was not inhibited, and its level was not different with that in the outer-petal, which converted upstream materials to downstream materials in large quantity. In the expression analysis of the 10 candidate DEGs, the expression of upstream 
PlCHI was also not inhibited in the inner-petal, instead its level was higher than that in the outer-petal, which was opposite to our previous results [30], indicating that chalcone was not accumulated in the inner-petal and did not cause its yellow colour. Furthermore, the expression levels of downstream PlDFR, PlANS, Pl3GT and Pl5GT in the inner-petal were all lower compared to the outer-petal, but this difference did not reach hundreds-fold as our previous results [30], suggesting that anthocyanins were also produced in the innerpetal, just its content was relatively low.

Flower colour was mainly dependent on the kinds of pigment, the content and its distribution in the petals [49]. The generalised yellow flower from pale yellow to dark yellow could be divided into multiple grades according to its colour depth. Among these colours, the pigment of the yellow flower perceived by the naked eye was relatively complicated, which was related to flavonoids and carotenoids, this conclusion had been fully proved by previous transcriptome studies [20-23]. Previous studies on the determination of $P$. lactiflora petal pigment composition showed that a great deal of chalcone and a small amount of anthoxanthin accumulation were the cause of its yellow petal formation $[27,30]$. Similarly, the biochemical mechanism of yellow characteristic formation in P. suffruticosa was the same, and kaempferol, quercetin, isorhamnetin, chrysoeriol, apigenin and luteolin were identified as the main anthoxanthins [50]. This was mainly because anthocyanins were responsible for red, whereas chalcones and anthoxanthins were yellow. In this study, chalcones could not be detected in the yellow inner-petal, whereas a large number of anthoxanthins (kaempferol di-hexoside, kaempferol-3-O-malonylglucoside7-O-glucoside, quercetin-3-O-galactoside, luteolin-7-Ogalloylglucoside, luteolin-7-O-glucoside, isorhamnetin-3$\mathrm{O}$-glucoside and flavone $\mathrm{C}$-glycoside) and a few anthocyanins (cyanidin-3,5-di-O-glucoside, peonidin-3, 5 -di-O-glucoside) were identified, which was not consistent with previous results $[27,30]$. Although anthocyanins were in the yellow inner-petal, the co-pigmentation effect of anthoxanthins in the inner-petal was significant and completely covered the red effect of anthocyanins due to the increase of CI from 34.00 in S1 to 69.28 in S4. In addition, compared with the red outer-petal, the contents of anthoxanthins and anthocyanins were relatively lower in the yellow inner-petal. These physiological results obtained perfect explanation using key gene expression analysis. Due to the massive expression of upstream PlPAL and PlCHI, particularly PlCHI, a large number of upstream materials in the inner-petal were formed and transformed to the downstream, rather than in the accumulation of chalcones. With the expression of $P l F 3 H$, PlF3' $H$, PlF3'5' $H$ and PlFLS, a variety of anthoxanthins was formed and accumulated. Furthermore, a small amount of anthocyanins was produced, which was combined with low expression levels of downstream PlDFR, PlANS, Pl3GT and Pl5GT, and eventually made the inner-petal appear yellow. In addition, because the expression level of the first key gene, PlPAL, in the inner-petal was always lower than that in the outer-petal, combined with lower PlFLS, PlDFR, PlANS, Pl3GT and Pl5GT expression, the accumulation of anthoxanthins and anthocyanins in the inner-petal was lower compared to the outer-petal. Taken together, these results provided an understanding of the biochemical and molecular mechanisms of yellow characteristic formation in P. lactiflora, which might lay a theoretical foundation for breeding new yellow P. lactiflora varieties.

\section{Conclusion}

This study showed that RNA-Seq analysis based on the high throughput sequencing technology provided an efficient approach for identifying critical genes in P. lactiflora. In the metabolic pathways related to yellow formation, flavonoid metabolic pathway and glucide metabolic pathway were identified, and PlPAL, PlFLS, PIDFR, PlANS, Pl3GT and Pl5GT were selected as potential candidates involved in flavonoid metabolic pathway, which induced inhibition of anthocyanin biosynthesis mediated yellow formation in P. lactiflora.

\section{Methods}

\section{Plant materials}

$P$. lactiflora was grown in the germplasm repository of Horticulture and Plant Protection College, Yangzhou University, Jiangsu Province, China (32 $\left.30^{\prime} \mathrm{N}, 119^{\circ} 25^{\prime} \mathrm{E}\right)$. A flower colour chimaera cultivar 'Jinhui' with red outerpetal and yellow inner-petal was used as the experimental materials, and the ground plants grew well with sufficient light and water supply. The samples were taken from March to May 2013, including four developmental stages (S1, flower-bud stage; S2, initiating bloom stage; S3, bloom stage; and S4, wither stage). Among these samples, the young outer-petal and inner-petal samples with significantly different colours were used for transcriptome sequencing. In addition, the developmental petals were used for qualitative and quantitative analysis of flavonoids and expression analysis of candidate genes. After measurement of the flower qualitative trait and colour parameter, all samples were immediately frozen in liquid nitrogen and stored at $-80^{\circ} \mathrm{C}$ until further analysis.

\section{Flower qualitative trait and colour variables measurements}

Flower diameter was measured by micrometer scale (Taizhou Xinshangliang Measuring Tools Co., Ltd., China). And the colour of fresh petals were compared with the RHSCC firstly [51], and then measured on a 
TC-P2A chroma meter (Beijing Optical Instrument Factory, China) using three colour parameters including $L^{*}, a^{*}$ and $b^{*}$ values.

\section{RNA extraction, cDNA library construction and transcriptome sequencing}

Total RNA was extracted according to a modified CTAB extraction protocol [25]. Prior to cDNA library construction, RNA samples were treated with DNase using DNase I kit (TaKaRa, Japan) based on the manufacturer's instructions, and then their integrity were examined by a spectrophotometer (Eppendorf, Germany) and 1\% agarose gel electrophoresis. After these, magnetic beads with Oligo (dT) were used to isolate mRNA which was fragmented into short fragments using a fragmentation buffer. Then the first cDNA strand was synthesized using the mRNA fragments as templates, and the second strand was generated using a SuperScript Double-Stranded cDNA Synthesis kit (Invitrogen, Camarillo, CA), purified via magnetic beads, the ends repaired and a single nucleotide A (adenine) added to the 3' ends. Sequencing adaptors were then ligated to the fragments, and the suitable fragments were selected for the PCR amplification as templates. After quantification and qualification of the sample library using the Agilent 2100 Bioanalyzer (Agilent Technologies, Palo Alto, CA) and ABI StepOnePlus Real-Time PCR System (Applied Biosystems, USA), the samples were sequenced at Beijing Genomic Institute (Shenzhen, China) using an Illumina HiSeq 2000 platform (Illumina Inc., San Diego, CA, USA), and the workflow was as follows: template hybridization, isothermal amplification, linearization, blocking, sequencing primer hybridization, and sequencing on the sequencer for read 1. After completion of the first read, the templates could be regenerated in situ to enable a second read from the opposite end of the fragments. Once the original templates were cleaved and removed, the reverse strands underwent sequencing-by-synthesis.

\section{De novo assembly}

After raw reads filtering, transcriptome de novo assembly was performed using the short reads assembling program Trinity, which combined three independent software modules: Inchworm, Chrysalis and Butterfly [12]. Firstly, Inchworm assembled the RNA-seq data into unique sequences of transcripts, which often generate full-length transcripts for a dominant isoform, but reported only the unique portions of alternatively spliced transcripts. Secondly, Chrysalis clustered the Inchworm Contigs into clusters and constructed complete de Bruijn graphs for each cluster, which represented the full transcriptional complexity for a given gene. Next, Chrysalis partitioned the full read set among these disjointed graphs. Subsequently, Butterfly processed the individual graphs in parallel, which traced the paths that reads and pairs of reads took within the graph, ultimately reporting full-length transcripts for alternatively spliced isoforms and teasing apart transcripts that corresponded to paralogous genes. The result sequences of Trinity were called Unigenes. Finally, blastx alignment (e-value $<0.00001)$ between Unigenes and NR (http://www.ncbi.nlm.nih.gov), Swiss-Prot (http://www.expasy.ch/sprot), KEGG (http:// www.genome.jp/kegg) and COG (http://www.ncbi.nlm. nih.gov/COG) was performed, and the best aligning results were used to determine the sequence direction of Unigenes. If the results of different databases conflicted with each other, then a priority order of NR, Swiss-Prot, KEGG and COG was given to determine the sequence direction of the Unigenes. When a Unigene was unaligned with any of these databases, a software named ESTScan was used to determine its sequence direction [52]. For Unigenes with sequence directions, we provided their sequences from the $5^{\prime}$ end to the $3^{\prime}$ end; for those without any direction, we provided their sequences from the assembly software.

\section{Unigene annotation and analysis}

Unigene annotation was performed using various bioinformatics procedures. In addition, the sequences were firstly aligned using blastx to protein databases such as NR, Swiss-Prot, KEGG and COG (e-value $<0.00001$ ), and aligned by blastn to NT (e-value $<0.00001)$, thereby retrieving proteins with the highest sequence similarity with the given Unigenes along with their protein functional annotations. Secondly, the Blast2GO program [53] was used to obtain the GO annotation of Unigenes according to the NR annotation. After obtaining the GO annotation for every Unigene, WEGO software [54] was used to perform the GO functional classification for all Unigenes and to understand the distribution of the gene functions of the species on the macro-level. In addition, using the KEGG database, the complex biological behaviours of genes were further studied and pathway annotation for Unigenes was obtained by KEGG annotation.

\section{Unigene expression difference analysis}

Unigene expression level was calculated using Fragments Per kb per Million fragments (FPKM) method [55]. DEGs were defined based on false discovery rate (FDR $\leq 0.001$ and differential expression fold $>2$ ). The confirmed DEGs were subjected to GO functional analysis and KEGG Pathway analysis.

\section{Gene expression analysis}

Gene transcript levels were analysed using Q-PCR with a BIO-RAD CFX96 ${ }^{\text {TM }}$ Real-Time System (Bio-Rad, USA). The cDNA was synthesized from $1 \mu \mathrm{g}$ RNA 
using PrimeScript ${ }^{\oplus}$ RT reagent Kit With gDNA Eraser (TaKaRa, Japan). P. lactiflora Actin (JN105299) had been used as an internal control in this study [56]. All gene-specific primers in this paper were showed in Additional file 5: Table S3. Q-PCR was performed using the SYBR ${ }^{\circ}$ Premix Ex Taq ${ }^{\text {Tix }}$ (Perfect Real Time) (TaKaRa, Japan). Gene relative expression levels of target genes were calculated by the $2^{-\triangle \triangle \mathrm{Ct}}$ comparative threshold cycle $(\mathrm{Ct})$ method [57], and the expression level of $P A L$ in S1 of inner-petal was used as the control. The Ct values of the triplicate reactions were gathered using the Bio-Rad CFX Manager V1.6.541.1028 software.

\section{Qualitative and quantitative analysis of flavonoids}

Flavonoid analysis was performed according to the method of Jia et al. [27] and He et al. [58] with some modifications. The petals of each sample $(1.0 \mathrm{~g}$ fresh weight) were extracted with $6 \mathrm{~mL}$ of acidic methanol solution (70: 0.1: 29.9; v/v/v, $\mathrm{CH}_{3} \mathrm{OH}: \mathrm{HCl}: \mathrm{H}_{2} \mathrm{O}$ ) in a test tube and shaken in a XW-80A vortex (Shanghai Huxi Analysis Instrument Factory Co., Ltd., China) at $4^{\circ} \mathrm{C}$ for $24 \mathrm{~h}$. The filtrate was passed through $0.22 \mu \mathrm{m}$ membrane filters (Shanghai ANPEL Scientific Instrument Co., Ltd., China). This experiment was performed three times for each sample, and the extracts were used for analysis of the flavonoids.

Qualitative analysis of the flavonoids was performed using HPLC-ESI-MS ${ }^{\mathrm{n}}$ (LCQ Deca XP MAX, Thermo). The HPLC column was TSK gel ODS-80Ts QA ( $4.6 \mathrm{~mm} \times$ $250 \mathrm{~mm}$ ) (Tosoh, Japan). The column temperature was $35^{\circ} \mathrm{C}$, and the injected volume was $20 \mu \mathrm{L}$. The flow rate was $0.8 \mathrm{~mL} / \mathrm{min}$, and the mobile phase consisted of $10 \%$ formic acid water solution as solvent A (10:90; v/v, $\mathrm{HCOOH}: \mathrm{H}_{2} \mathrm{O}$ ) and methyl alcohol/acetonitrile/water (10:40:50; v/v/v, $\mathrm{CH}_{3} \mathrm{OH}: \mathrm{CH}_{3} \mathrm{CN}: \mathrm{H}_{2} \mathrm{O}$ ) as solvent B. The linear gradient profile was $10 \% \mathrm{~B}$ at $0 \mathrm{~min}, 20 \% \mathrm{~B}$ at $30 \mathrm{~min}, 30 \% \mathrm{~B}$ at $50 \mathrm{~min}, 40 \% \mathrm{~B}$ at $60 \mathrm{~min}, 50 \% \mathrm{~B}$ at $65 \mathrm{~min}, 50 \% \mathrm{~B}$ at $70 \mathrm{~min}, 10 \% \mathrm{~B}$ at $75 \mathrm{~min}$, and then returned to $10 \%$ B in 90 min. Analysis conditions of mass spectrometry were as follows: positive ionization mode, gas $\left(\mathrm{N}_{2}\right)$ temperature, $300^{\circ} \mathrm{C}$; nebulizer pressure, $45 \mathrm{~Pa}$; sheath gas temperature, $250^{\circ} \mathrm{C}$; capillary voltage, $3.5 \mathrm{kV}$; nozzle voltage, $500 \mathrm{~V}$; and scan range, $\mathrm{m} / \mathrm{z} 50-$ 1500 units.

Quantitative analysis of the flavonoids was performed on an HPLC (Agilent 1200-6460 QQQ, USA) equipped with a Quat Pump, thermostatted Column Compartment 1260 TCC and a Diode Array Detector 1260 DAD. The chromatography analysis conditions were consistent with that of mass spectrometry. Detection was performed at $525 \mathrm{~nm}$ for anthocyanins, $350 \mathrm{~nm}$ for anthoxanthins (flavone and flavonol) and $365 \mathrm{~nm}$ for chalcones, and the photodiode array spectra were recorded between 200 and $800 \mathrm{~nm}$. All determinations were performed with three replicates. The amount of total anthocyanins and anthoxanthins was calculated in milligrams per gram fresh weight (as a quantity of Malvidin-3,5-di-O-glucoside (Mv3G5G) $\mathrm{mg} \mathrm{g}^{-1}$ and as a quantity of Rutin $\mathrm{mg} \mathrm{g}^{-1}$, respectively).

\section{Additional files}

Additional file 1: Figure S1. Randomicity of P. lactiflora outer-petal and inner-petal reads on All-Unigene.

Additional file 2: Figure S2. Figures of NR annotation E-value, similarity and species distribution statistics.

Additional file 3: Table S1. List of pathway enriched differentially expressed genes in P. lactiflora outer-petal and inner-petal.

Additional file 4: Table S2. Identification of pigment components in P. lactiflora petals [59-62].

Additional file 5: Table S3. Gene-specific primers sequence for detection by Q-PCR.

\section{Abbreviations}

3GT: Anthocyanidin 3-O-glucosyltransferase gene; 5GT: Anthocyanidin 5-O-glucosyltransferase gene; ANS: Anthocyanidin synthase gene;

CHI: Chalcone isomerase gene; CHS: Chalcone synthase gene; Cl: Co-pigment index; COG: Cluster of orthologous groups of proteins database;

Ct: Threshold cycle; DEGs: Differentially expressed genes;

DFR: Dihydroflavonol 4-reductase gene; F3H: Flavanone 3-hydroxylase gene; $\mathrm{F3}^{\prime} \mathrm{H}$ : Flavonoid $3^{\prime}$-hydroxylase gene; $\mathrm{F3}^{\prime} 5^{\prime} \mathrm{H}$ : Flavonoid $3^{\prime}, 5^{\prime}$ - hydroxylase gene; FLS: Flavonol synthase gene; FPKM: Fragments per kb per million fragments; GO: Gene ontology database; HPLC-ESI-MS ${ }^{n}$ : High-performance liquid chromatograph-electrospray ionization-mass spectrometry;

KEGG: Kyoto encyclopedia of genes and genomes database; MT: Methyl transferase gene; NR: Non-redundant protein database; NT: Nucleotide databases; PAL: Phenylalanine ammonialyase gene; RHSCC: Royal horticultural society colour chart; RNA-Seq: Transcriptome sequencing; Swiss-Prot: Swiss-Prot protein database.

\section{Competing interests}

The authors declare that they have no competing interests.

\section{Authors' contributions}

DZ and JT conceived the study and designed the experiments. DZ, YJ, CN, JM, SL and WD performed the experiments. DZ analyzed the data and wrote the manuscript with suggestions by JT. All authors read and approved the final manuscript.

\section{Acknowledgments}

This work was supported by the Natural Science Foundation of China (31372097), the Major Project of College Natural Science Research of Jiangsu Province (13KJA210005), the Priority Academic Program Development from Jiangsu Government and 2013 College Academic Science and Technology Innovation Fund of Yangzhou University for Student. And we thank Dr. Yanyan Zhang from Testing Center, Yangzhou University for technical assistance on qualitative and quantitative analysis of flavonoids.

Received: 23 April 2014 Accepted: 15 August 2014 Published: 19 August 2014

\section{References}

1. Nishihara M, Nakatsuka T: Genetic engineering of flavonoid pigments to modify flower color in floricultural plants. Biotechnol Lett 2011, 33:433-441.

2. Kalba LA: Blue roses and yellow violets: flowers and the cultivation of color in nineteenth-century France. Representations 2012, 120:83-114.

3. Zhao D, Hao Z, Wang J, Tao J: Effects of $\mathrm{pH}$ in irrigation water on plant growth and flower quality in herbaceous peony (Paeonia lactiflora Pall.). Sci Hortic 2013, 154:45-53. 
4. Wang J, Zhang Z: Herbaceous Peonies of China. Beijing: China Forestry Publishing House; 2005.

5. Wu Z: Studies on Resource Exploration and Appraisable Application of Forcing Culture for Chinese Cultiver Groups of Paenoia lactiflora, Master's thesis. Beijing Forestry University; 2006

6. Wang Z, Gerstein M, Snyder M: RNA-Seq: a revolutionary tool for transcriptomics. Nat Rev Genet 2009, 10:57-63.

7. Marioni JC, Mason CE, Mane SM, Stephens M, Gilad Y: RNA-seq: an assessment of technical reproducibility and comparison with gene expression arrays. Genome Res 2008, 18:1509-1517.

8. Fullwood MJ, Wei CL, Liu ET, Ruan Y: Next-generation DNA sequencing of paired-end tags (PET) for transcriptome and genome analyses. Genome Res 2009, 19:521-532.

9. Hoeijmakers WA, Bártfai R, Stunnenberg HG: Transcriptome analysis using RNA-Seq. Methods Mol Biol 2013, 923:221-239.

10. Vera JC, Wheat CW, Fescemyer HW, Frilander MJ, Crawford DL, Hanski I, Marden JH: Rapid transcriptome characterization for a nonmodel organism using 454 pyrosequencing. Mol Ecol 2008, 17:1636-1647.

11. Brautigam A, Mullick T, Schliesky S, Weber APM: Critical assessment of assembly strategies for non-model species mRNA-Seq data and application of next-generation sequencing to the comparison of C (3) and C (4) species. J Exp Bot 2011, 62:3093-3102.

12. Grabherr MG, Haas BJ, Yassour M, Levin JZ, Thompson DA, Amit I, Adiconis X, Fan L, Raychowdhury R, Zeng Q, Chen Z, Mauceli E, Hacohen N, Gnirke A, Rhind N, di Palma F, Birren BW, Nusbaum C, Lindblad-Toh K, Friedman N, Regev A: Full-length transcriptome assembly from RNA-Seq data without a reference genome. Nat Biotechnol 2011, 29:644-652

13. Qi YX, Liu YB, Rong WH: RNA-Seq and its applications: a new technology for transcriptomics. Hereditas 2011, 33:1191-1202.

14. Guo S, Zheng Y, Joung JG, Liu S, Zhang Z, Crasta OR, Sobral BW, Xu Y, Huang S, Fei Z: Transcriptome sequencing and comparative analysis of cucumber flowers with different sex types. BMC Genomics 2010, 11:384.

15. Zhang N, Zhang HJ, Zhao B, Sun QQ, Cao YY, Li R, Wu XX, Weeda S, Li L, Ren S, Reiter RJ, Guo YD: The RNA-seq approach to discriminate gene expression profiles in response to melatonin on cucumber lateral root formation. J Pineal Res 2014, 56:39-50.

16. Tong C, Wang X, Yu J, Wu J, Li W, Huang J, Dong C, Hua W, Liu S: Comprehensive analysis of RNA-seq data reveals the complexity of the transcriptome in Brassica rapa. BMC Genomics 2013, 14:689.

17. Rowland LJ, Alkharouf N, Darwish O, Ogden EL, Polashock JJ, Bassil NV, Main $D$ : Generation and analysis of blueberry transcriptome sequences from leaves, developing fruit, and flower buds from cold acclimation through deacclimation. BMC Plant Biol 2012, 12:46.

18. Wang L, Zhao S, Gu C, Zhou Y, Zhou H, Ma J, Cheng J, Han Y: Deep RNA-Seq uncovers the peach transcriptome landscape. Plant Mol Biol 2013, 8:365-377.

19. Liang C, Liu X, Yiu SM, Lim BL: De novo assembly and characterization of Camelina sativa transcriptome by paired-end sequencing. BMC Genomics 2013, 14:146.

20. Yu K, Xu Q, Da X, Guo F, Ding Y, Deng X: Transcriptome changes during fruit development and ripening of sweet orange (Citrus sinensis). BMC Genomics 2012, 13:10.

21. Feng C, Chen M, Xu CJ, Bai L, Yin X, Li X, Allan AC, Ferguson IB, Chen K: Transcriptomic analysis of Chinese bayberry (Myrica rubra) fruit development and ripening using RNA-Seq. BMC Genomics 2012, 13:19.

22. Yang $N$, Zhao K, Chen L: Deep sequencing-based transcriptome profiling analysis of Chimonanthus praecox reveals insights into secondary metabolites biosynthesis. J Beijing Forestry Univ 2012, 34:104-107.

23. Huang $L$, Yang $X$, Sun $P$, Tong W, Hu S: The first Illumina-based De Novo transcriptome sequencing and analysis of safflower flowers. PLOS ONE 2012, 7:38653.

24. Himi E, Maekawa M, Noda K: Differential expression of three flavanone 3-hydroxylase genes in grains and coleoptiles of wheat. Int J Plant Genom 2011, 2011:1-11

25. Zhao D, Zhou C, Tao J: Carotenoid accumulation and carotenogenic genes expression during two types of persimmon fruit (Diospyros kaki L.) development. Plant Mol Biol Report 2011, 29:646-654.

26. Ogata H, Goto S, Sato K, Fujibuchi W, Bono H, Kanehisa M: KEGG: Kyoto encyclopedia of genes and genomes. Nucleic Acids Res 1999, 27:29-34

27. Jia N, Shu Q, Wang L, Du H, Xu Y, Liu Z: Analysis of petal anthocyanins to investigate coloration mechanism in herbaceous peony cultivars. Sci Hortic 2008, 117:167-173.
28. Jia N, Shu Q, Wang D, Wang L, Liu Z, Ren H: Identification and characterization of anthocyanins by high-performance liquid chromatography-electrospray ionization-mass spectrometry in herbaceous peony species. J Am Soc Hortic Sci 2008, 133:418-426.

29. Zhong P, Wang L, Li S, Xu Y, Zhu M: The changes of floral color and pigments composition during the flowering period in Paeonia lactiflora Pallas. Acta Horticulturae Sinica 2012, 39:2271-2282.

30. Zhao D, Tao J, Han C, Ge J: Flower color diversity revealed by differential expression of flavonoid biosynthetic genes and flavonoid accumulation in herbaceous peony (Paeonia lactiflora Pall.). Mol Biol Rep 2012, 39:11263-11275.

31. Nakatsuka A, Mizuta D, Kii Y, Miyajima I, Kobayashi N: Isolation and expression analysis of flavonoid biosynthesis genes in evergreen azalea. Sci Hortic 2008, 118:314-320.

32. Tan J, Wang $M, T u L$, Nie $Y$, Lin $Y$, Zhang $X$ : The flavonoid pathway regulates the petal colors of cotton flower. PLOS ONE 2013, 8:e72364

33. Tang Q, Ma X, Mo C, Wilson IW, Song C, Zhao H, Yang Y, Fu W, Qiu D: An efficient approach to finding Siraitia grosvenorii triterpene biosynthetic genes by RNA-seq and digital gene expression analysis. BMC Genomics 2011, 12:343.

34. Kaul S, Koo HL, Jenkins J, Rizzo M, Rooney T, Tallon LJ, Feldblyum T, Nierman W, Benito MI, Lin XY, Town CD, Venter JC, Fraser CM, Tabata S, Nakamura Y, Kaneko T, Sato S, Asamizu E, Kato T, Kotani H, Sasamoto S, Ecker JR, Theologis A, Federspiel NA, Palm CJ, Osborne BI, Shinn P, Conway $A B$, Vysotskaia VS, Dewar K, et al: Analysis of the genome sequence of the flowering plant Arabidopsis thaliana. Nature 2000, 408:796-815.

35. Zhang Q, Chen W, Sun L, Zhao F, Huang B, Yang W, Tao Y, Wang J, Yuan Z, Fan G, Xing Z, Han C, Pan H, Zhong X, Shi W, Liang X, Du D, Sun F, Xu Z, Hao R, Lv T, Lv Y, Zheng Z, Sun M, Luo L, Cai M, Gao Y, Wang J, Yin Y, Xu X, et al: The genome of Prunus mume. Nat Commun 2012, 3:1318.

36. Ming R, VanBuren R, Liu Y, Yang M, Han Y, Li LT, Zhang Q, Kim MJ, Schatz MC, Campbell M, Li J, Bowers JE, Tang H, Lyons E, Ferguson AA, Narzisi G, Nelson DR, Blaby-Haas CE, Gschwend AR, Jiao Y, Der JP, Zeng F, Han J, Min XJ, Hudson KA, Singh R, Grennan AK, Karpowicz SJ, Watling JR, Ito K, et al: Genome of the long-living sacred lotus (Nelumbo nucifera Gaertn.). Genome Biol 2013, 14:R41.

37. Cheng S, van den Bergh E, Zeng P, Zhong X, Xu J, Liu X, Hofberger J, de Bruijn S, Bhide AS, Kuelahoglu C, Bian C, Chen J, Fan G, Kaufmann K, Hall JC, Becker A, Bräutigam A, Weber AP, Shi C, Zheng Z, Li W, Lv M, Tao Y, Wang J, Zou H, Quan Z, Hibberd JM, Zhang G, Zhu XG, Xu X, et al: The Tarenaya hassleriana genome provides insight into reproductive trait and genome evolution of crucifers. Plant Cell 2013, 25:2813-2830.

38. Yagi M, Kosugi S, Hirakawa H, Ohmiya A, Tanase $\mathrm{K}$, Harada T, Kishimoto $\mathrm{K}$, Nakayama M, Ichimura K, Onozaki T, Yamaguchi H, Sasaki N, Miyahara T, Nishizaki Y, Ozeki Y, Nakamura N, Suzuki T, Tanaka Y, Sato S, Shirasawa K, Isobe S, Miyamura Y, Watanabe A, Nakayama S, Kishida Y, Kohara M, Tabata S: Sequence analysis of the genome of carnation (Dianthus caryophyllus L.). DNA Res 2013. online, doi:10.1093/dnares/dst053.

39. Velculescu VE, Zhang L, Zhou W, Vogelstein J, Basrai MA, Bassett DE Jr, Hieter P, Vogelstein B, Kinzler KW: Characterization of the yeast transcriptome. Cell 1997, 88:243-251.

40. De Cremer K, Mathys J, Vos C, Froenicke L, Michelmore RW, Cammue BPA, De Coninck B: RNAseq-based transcriptome analysis of Lactuca sativa infected by the fungal necrotroph Botrytis cinerea. Plant Cell Environ 2013, 36:1992-2007.

41. Tang X, Tang Z, Huang S, Liu J, Liu J, Shi W, Tian X, Li Y, Zhang D, Yang J, Gao Y, Zeng D, Hou P, Niu X, Cao Y, Li G, Li X, Xiao F, Liu Y: Whole transcriptome sequencing reveals genes involved in plastid/chloroplast division and development are regulated by the HP1/DDB1 at an early stage of tomato fruit development. Planta 2013, 238:923-936.

42. Yang $L$, Ding $G$, Lin $H$, Cheng $H$, Kong $Y$, Wei $Y$, Fang $X$, Liu R, Wang $L$, Chen $X$, Yang C: Transcriptome analysis of medicinal plant Salvia miltiorrhiza and identification of genes related to tanshinone biosynthesis. PLOS ONE 2013, 8:e80464.

43. Garg R, Patel RK, Jhanwar S, Priya P, Bhattacharjee A, Yadav G, Bhatia S, Chattopadhyay D, Tyagi AK, Jain M: Gene discovery and tissue-specific transcriptome analysis in chickpea with massively parallel pyrosequencing and web resource development. Plant Physiol 2011, 156:1661-1678.

44. Wang Y, Zeng X, lyer NJ, Bryant DW, Mockler TC, Mahalingam R: Exploring the switchgrass transcriptome using second-generation sequencing technology. PLOS ONE 2012, 7:e34225. 
45. Wang Y, Xu L, Chen Y, Shen H, Gong Y, Limera C, Liu L: Transcriptome profiling of radish (Raphanus sativus $L$.) root and identification of genes involved in response to lead $(\mathrm{Pb})$ stress with next generation sequencing. PLOS ONE 2013, 8:e66539.

46. Zhang Y, Zhu J, Dai H: Characterization of transcriptional differences between columnar and standard apple trees using RNA-Seq. Plant Mol Biol Report 2012, 30:957-965.

47. Xu Q, Yu K, Zhu A, Ye J, Liu Q, Zhang J, Deng X: Comparative transcripts profiling reveals new insight into molecular processes regulating lycopene accumulation in a sweet orange (Citrus sinensis) red-flesh mutant. BMC Genomics 2009, 10:540.

48. Lu X, Zhou W, Gao F: Cloning, characterization and localization of CHS gene from blood orange, Citrus sinensis (L.) Osbeck cv. Ruby. Mol Biol Rep 2009, 36:1983-1990.

49. Miller R, Owens SJ, Rorslett B: Plants and colour: flowers and pollination. Optic Laser Tech 2011, 43:282-294.

50. Li C, Du H, Wang L, Shu Q, Zheng Y, Xu Y, Zhang J, Zhang J, Yang R, Ge Y: Flavonoid composition and antioxidant activity of tree peony (Paeonia section Moutan) yellow flowers. J Agric Food Chem 2009, 57:8496-8503.

51. Hu SY: The tour of a botanist in China. Arnoldia 1976, 35:264-295.

52. Iseli C, Jongeneel CV, Bucher P: ESTScan: a program for detecting, evaluating, and reconstructing potential coding regions in EST sequences. Proceedings of the International Conference on Intelligent Systems for Molecular Biology 1999, 99:138-148.

53. Conesa A, Gotz S, Carcia JM, Terol J, Talon M, Robles M: Blast2GO: a universal tool for annotation, visualization and analysis in functional genomics research. Bioinformatics 2005, 21:3674-3676.

54. Ye J, Fang L, Zheng H, Zhang Y, Chen J, Zhang Z, Wang J, Li S, Li R, Bolund L, Wang J: WEGO: a web tool for plotting GO annotations. Nucleic Acids Res 2006, 34:293-297.

55. Mortazavi A, Williams BA, McCue K, Schaeffer L, Wold B: Lorian schaeffer and barbara wold mapping and quantifying mammalian transcriptomes by RNA-Seq. Nat Methods 2008, 5:621-628.

56. Zhao D, Tao J, Han C, Ge J: Actin as an alternative internal control gene for gene expression analysis in herbaceous peony (Paeonia lactiflora Pall.). Afr J Agric Res 2012, 7:2153-2159.

57. Schmittgen TD, Livak KJ: Analyzing real-time PCR data by the comparative CT method. Nat Protoc 2008, 36:1101-1108.

58. He Q, Shen $Y$, Wang M, Huang M, Yang R, Zhu S, Wang L, Xu Y, Wu R. Natural variation in petal color in Lycoris longituba revealed by anthocyanin components. PLOS ONE 2011, 6:e22098.

59. Fan J, Zhu W, Kang H, Ma H, Tao G: Flavonoid constituents and antioxidant capacity in flowers of different Zhongyuan tree penoy cultivars. J Funct Foods 2012, 4:147-157.

60. Chen S, Fang L, Xi H, Guan L, Fang J, Liu Y, Wu B, Li S: Simultaneous qualitative assessment and quantitative analysis of flavonoids in various tissues of lotus (Nelumbo nucifera) using high performance liquid chromatography coupled with triple quad mass spectrometry. Anal Chim Acta 2012, 724:127-135.

61. Tuberosoa CIG, Montorob P, Piacente S, Corona G, Deiana M, Dessì MA, Pizza C, Cabras P: Flavonoid characterization and antioxidant activity of hydroalcoholic extracts from Achillea ligustica All. J Pharm Biomed Anal 2009, 50:440-448.

62. Rui W, Feng Y, Liu S, Tan Y: Analysis of flavonoids in Ranunculus Japonicus by UPLC HILIC/Q-TOF-MS. Asia-Pacific Traditional Medicine 2011, 7:18-21.

doi:10.1186/1471-2164-15-689

Cite this article as: Zhao et al:: Transcriptome sequencing of a chimaera reveals coordinated expression of anthocyanin biosynthetic genes mediating yellow formation in herbaceous peony (Paeonia lactiflora Pall.). BMC Genomics 2014 15:689.

\section{Submit your next manuscript to BioMed Central and take full advantage of:}

- Convenient online submission

- Thorough peer review

- No space constraints or color figure charges

- Immediate publication on acceptance

- Inclusion in PubMed, CAS, Scopus and Google Scholar

- Research which is freely available for redistribution

Submit your manuscript at www.biomedcentral.com/submit 